\title{
SEPARABILITY IN THE STRICT AND SUBSTRICT TOPOLOGIES
}

\author{
W. H. SUMMERS ${ }^{1}$
}

\begin{abstract}
Let $X$ denote a locally compact Hausdorff space, $C_{b}(X)$ the space of all bounded continuous complex valued functions on $X$, and $\beta$ the strict topology for $C_{b}(X)$. The separability of $\left(C_{b}(X), \beta\right)$ is characterized in terms of $X$, albeit in a more general context. This result provides a negative answer to a conjecture made by Todd, contains the classical separability theorems for continuous function spaces, and relates to the concepts of $\tau$-smooth and tight functionals.
\end{abstract}

Introduction. In 1958, R. C. Buck [1] introduced a locally convex topology on the space $C_{b}(X)$ of bounded continuous functions on a locally compact Hausdorff space $X$ which he called the strict topology, and established that the space of strictly continuous linear functionals on $C_{b}(X)$ was precisely the space $M_{b}(X)$ of bounded Radon measures on $X$. Subsequently, A. C. M. van Rooij [11] and F. D. Sentilles [13] have enhanced the role of the strict topology by introducing extensions to the completely regular case which give rise to the dual spaces $\mathscr{M}_{t}(X)$ and $\mathscr{M}_{\tau}(X)$ of tight and $\tau$-smooth linear functionals, respectively.

The spaces $\mathscr{M}_{t}(X)$ and $\mathscr{M}_{\tau}(X)$, each endowed with the weak topology from its pairing with $C_{b}(X)$ where $X$ now denotes a completely regular Hausdorff space, have already been the subject of intensive study (e.g., see [9], [17]), and the relationship between the metrizability of certain subsets of these spaces and the metrizability of $X$ (see Varadarajan [17]) leads naturally to a consideration of separability in the aforementioned extensions of the strict topology. The purpose of our note is to begin such a study.

1. Preliminaries. In what follows, $X$ will denote a completely regular Hausdorff space, while all functions will be complex valued unless it is

Presented to the Society, January 18, 1972; received by the editors December 20, 1971 and, in revised form, February 22, 1972.

AMS 1970 subject classifications. Primary 46E10; Secondary 28A30.

Key words and phrases. Strict topology, separable space, tight functional, $\tau$-smooth functional, submetrizable space.

1 This research was supported in part by National Science Foundation grant GP11762. 
explicitly stated otherwise. We shall let $C(X)$ denote the space of all continuous functions on $X$, let $B(X)$ denote the space of all bounded functions on $X$, and let $B_{0}(X)$ denote the subalgebra of $B(X)$ consisting of those elements in $B(X)$ which vanish at infinity. Further, let $C_{b}(X)=$ $C(X) \cap B(X)$ and $C_{0}(X)=C(X) \cap B_{0}(X)$.

For $f \in B(X),\|f\|=\sup \{|f(x)|: x \in X\}$ defines a norm on $B(X)$ inducing the uniform (convergence) topology, and if $\phi$ is a function on $X$ for which $\left\{f \phi: f \in C_{b}(X)\right\} \subseteq B(X)$, then $p_{\phi}(f)=\|f \phi\|$ defines a seminorm on $C_{b}(X)$. Assuming $X$ to be locally compact, Buck [1] defined the strict topology on $C_{b}(X)$ to be that locally convex topology generated by the set of seminorms $\left\{p_{\phi}: \phi \in C_{0}(X)\right\}$. Now $C_{0}(X)$ can be trivial when $X$ is not locally compact, but it is still possible to obtain useful generalizations of the strict topology in the completely regular case.

One such generalization is provided by the locally convex Hausdorff topology on $C_{b}(X)$ generated by the set of seminorms $\left\{p_{\phi}: \phi \in B_{0}(X)\right\}$. This topology, agreeing with the strict topology in the locally compact case, was introduced by van Rooij [11], and has since been considered under various equivalent formulations by the author [15], Sentilles [13], and Giles [4]. Following Sentilles, we will refer to this topology as the substrict topology and denote it by $\beta_{0}$.

The second generalization of the strict topology we will consider was introduced by Sentilles [13]. To define this topology, first observe that if $\beta X$ is the Stone-Cech compactification of $X$ and if $Q$ is a compact subset of $\beta X \mid X$, then $X_{Q}=\beta X \mid Q$ is locally compact and $C_{b}\left(X_{Q}\right)$ can be given the strict topology $\beta_{Q}$ generated by the set of seminorms $\left\{p_{\phi}: \phi \in C_{0}\left(X_{Q}\right)\right\}$. Using the restriction mappings from $C_{b}\left(X_{Q}\right)$ onto $C_{b}(X)$, the inductive limit topology on $C_{b}(X)$ induced by the topologies $\beta_{Q}$ taken over the collection $\mathscr{Q}$ of all compact subsets $Q$ of $\beta X \backslash X[10$, p. 79] is easily seen to agree with Buck's original strict topology in the locally compact case. Again following Sentilles, we will call this topology the strict topology and denote it by $\beta$.

Finally, we will need the following topological concepts in the sequel. A topological space $Y$ is called submetrizable if it can be mapped by a one-to-one continuous function onto some metric space $Z$. If, in addition, we can choose $Z$ to be separable, then we will say that $Y$ is separably submetrizable.

2. Separability in the substrict topology. Both Collins [2, p. 368] and Todd [16, p. 655] have obtained results concerning the separability of $\left(C_{b}(X), \beta_{0}\right)$ in the locally compact case, but even in this case there is apparently no characterization in terms of $X$. Todd [16, p. 656] does conjecture, however, that if $X$ is locally compact, then $\left(C_{b}(X), \beta_{0}\right)$ is separable 
if and only if $X$ is a separable metric space. Assuming only that $X$ is a completely regular Hausdorff space, we have the following characterization of separability in the substrict topology.

2.1. THEOREM. $\left(C_{b}(X), \beta_{0}\right)$ is separable if and only if $X$ is separably submetrizable.

Proof. For necessity, a standard argument prevails; namely, if $\left\{f_{n}\right\}_{n=1}^{\infty}$ is $\beta_{0}$ dense in $C_{b}(X)$, then the evaluation map $e$ on $X$ defined by $e(x)=\left(f_{n}(x)\right)_{n=1}^{\infty}$ is a one-to-one continuous function from $X$ into a separable metric space. Conversely, if $X$ is separably submetrizable, then there exists a metric space $Y$ with a countable dense subset $\left\{y_{n}\right\}_{n=1}^{\infty}$ and a one-to-one continuous function $h$ mapping $X$ onto $Y$. Moreover, we can assume that the topology on $Y$ is determined by a bounded metric $d$, and so the functions $\left\{f_{n}\right\}_{n=0}^{\infty}$ defined for each $x \in X$ by $f_{0}(x)=1$ and $f_{n}(x)=$ $d\left(h(x), y_{n}\right), n=1,2, \cdots$, all belong to $C_{b}(X)$. Now let $A$ be the smallest subalgebra of $C_{b}(X)$ which contains $\left\{f_{n}\right\}_{n=0}^{\infty}$. In order to show that $A$ is $\beta_{0}$ dense in $C_{b}(X)$ from whence the desired conclusion will follow, we first note that $\left(C_{b}(X), \beta_{0}\right)$ is a weighted space [15, p. 92], and $A$ is a priori a linear subspace of $C_{b}(X)$ which is an $A$-module. Consequently, we have an instance of the bounded case of the weighted approximation problem (see $[15$, p. 91]) which is also an instance of the separating case (see $\left[15\right.$, p. 96]) since the real functions $\left\{f_{n}\right\}_{n=1}^{\infty}$ clearly separate the points of $X$. Furthermore, $f_{0}(x) \neq 0$ for each $x \in X$, and hence our Stone-Weierstrass type theorem for weighted spaces [15, p. 97] implies $A$ is $\beta_{0}$ dense in $C_{b}(X)$.

It is interesting to note that the separability of $\left(C_{b}(X), \beta_{0}\right)$ is equivalent to the separability of $C(X)$ endowed with the compact-open topology $\kappa$.

2.2. Corollary (WARner [18, p. 270]). $(C(X), \kappa)$ is separable if and only if $X$ is separably submetrizable.

Proof. It is immediate from Theorem 2.1 that $(C(X), \kappa)$ is separable whenever $X$ is separably submetrizable, while replacing the $\beta_{0}$ dense sequence by a $\kappa$ dense sequence in the necessity argument used above yields the converse.

A proof of Theorem 2.1 could have been based on Warner's result (Corollary 2.2) by using van Rooij's characterization of the space $\left(C_{b}(X), \beta_{0}\right)^{*}$ of $\beta_{0}$ continuous linear functionals on $C_{b}(X)[11$, p. 41$]$. However, Warner's proof depended on the following classical result (e.g., [18, p. 265]) which can now be deduced from Theorem 2.1.

2.3. Theorem. $\left(C_{b}(X),\|\cdot\|\right)$ is separable if and only if $X$ is a compact metric space. 
Proof. First observe that $\beta_{0}$ agrees with the uniform topology on $C_{b}(X)$ whenever $X$ is compact. Consequently, if $X$ is a compact metric space, then it follows from Theorem 2.1 that $\left(C_{b}(X),\|\cdot\|\right)$ is separable. Conversely, if $\left(C_{b}(X),\|\cdot\|\right)$ is separable, then $\left(C_{b}(\beta X),\|\cdot\|\right)$ is separable. Theorem 2.1 now implies $\beta X$ is submetrizable and hence metrizable. Therefore, $\beta X=X[5, \mathrm{p} .132]$ and the proof is complete.

The well-known analogue [12, p. 140] of Theorem 2.3 for the space $\left(C_{0}(X),\|\cdot\|\right)$ can also be obtained from Theorem 2.1 , and in a somewhat strengthened form.

2.4. THEOREM. If $X$ is locally compact, then $\left(C_{0}(X),\|\cdot\|\right)$ is separable if and only if $X$ is a $\sigma$-compact metric space.

Proof. If $X$ is a $\sigma$-compact metric space, then its one-point compactification $X^{*}$ is metrizable. Therefore $\left(C_{b}\left(X^{*}\right),\|\cdot\|\right)$ is separable by Theorem 2.3 , and the separability of $\left(C_{0}(X),\|\cdot\|\right)$ is now immediate. For the converse, if $\left(C_{0}(X),\|\cdot\|\right)$ is separable, then there is a countable set $\left\{\phi_{n}\right\}_{n=1}^{\infty}$ contained in $C_{0}(X)$ which is $\beta_{0}$ dense in $C_{b}(X)$, whence $X$ is $\sigma$-compact [2, p. 368]. Since $X$ is submetrizable by Theorem $2.1, X$ is also locally metrizable, and so the metrizability of $X$ follows from Smirnov's metrization theorem (e.g., [6, p. 81]).

Using Smirnov's theorem to better advantage, the above proof actually establishes the following result.

2.5. THEOREM. Assume $X$ is both locally compact and paracompact. If $\left(C_{b}(X), \beta\right)$ is separable, then $X$ is metrizable.

2.6. COROLlaRY (TODD [16, p. 655]). If $X$ is both locally compact and $\sigma$-compact, then $\left(C_{b}(X), \beta\right)$ is separable if and only if $X$ is metrizable.

Proof. The necessity follows from Theorem 2.5 , while the sufficiency follows from Theorem 2.1.

Not only does Theorem 2.1 essentially contain the known results, it also provides a negative answer to the previously mentioned conjecture made by Todd. This can easily be seen by considering the real line $\boldsymbol{R}$ with the discrete topology. Unfortunately, it is not even true in the locally compact case that the separability of $\left(C_{b}(X), \beta_{0}\right)$ implies the metrizability of $X$. Indeed, Dieudonné [3] has given an example of a locally compact and separably submetrizable space which fails to be normal, and so it seems unlikely that the paracompactness hypothesis in Theorem 2.5 can be significantly weakened. Separably submetrizable spaces are not entirely devoid of nice properties nor unconnected with metrizable spaces, however, as we show in the next section. 
3. Separably submetrizable spaces. If $X$ is separably submetrizable, then every subspace of $X$ is also separably submetrizable. Furthermore, every subspace of $X$ is necessarily realcompact [5, p. 122]. Consequently, the only pseudocompact subspaces of a separably submetrizable space are the compact ones. In view of Theorem 2.1, these observations can be summarized in the following manner.

3.1. TheOrem. Assume $\left(C_{b}(X), \beta_{0}\right)$ is separable. If $Y$ is any subspace of $X$, then (1) $\left(C_{b}(Y), \beta_{0}\right)$ is separable, (2) $Y$ is realcompact, and (3) $Y$ is pseudocompact if and only if $Y$ is compact.

Of course, the cardinality of a separably submetrizable space is not greater than that of the continuum, but the converse is false as the preceding remarks plainly show when applied to the set of ordinals less than the first uncountable ordinal with the usual order topology. With some restrictions, however, this condition does become sufficient, and we have the following substantial improvement of Warner's result [18, p. 270] for discrete spaces. The cardinality of a set $Y$ will be denoted by $\operatorname{card}(Y)$.

3.2. THEOREM. If $X$ is metrizable, then $\left(C_{b}(X), \beta_{0}\right)$ is separable if and only if $\operatorname{card}(X) \leqq \operatorname{card}(\boldsymbol{R})$.

Proof. In view of Theorem 2.1 and our previous remarks, it will suffice to establish that if $\operatorname{card}(X) \leqq \operatorname{card}(\boldsymbol{R})$, then there exists a countable subset of $C(X)$ which distinguishes the points of $X$. To do this, let us first choose a one-to-one function $f$ mapping $X$ into $\boldsymbol{R}$. Now the topology for $X$ has a $\sigma$-discrete base [7, p. 127], and so for each natural number $n$ there is an index set $A_{n}$ and a discrete collection $\mathscr{B}_{n}=\left\{B_{n \alpha}: \alpha \in A_{n}\right\}$ of nonvoid open subsets of $X$ with $\mathscr{B}=\bigcup_{n=1}^{\infty} \mathscr{B}_{n}$ being a base for the topology. For each $n$ and for each $\alpha \in A_{n}$, choose $x_{n \alpha} \in B_{n \alpha}$. Further, since every closed set in $X$ is a zero set, for each $n$ and each $\alpha \in A_{n}$ there exists a $\phi_{n \alpha} \in C^{+}(X)$ such that $\phi_{n \alpha}^{-1}(0)=X \backslash B_{n \alpha}$. It is now easy to see that the functions

$$
f_{n}=\sum_{\alpha \in A_{n}} \phi_{n \alpha}, \quad g_{n}=\sum_{\alpha \in A_{n}} f\left(x_{n \alpha}\right) \phi_{n \alpha}
$$

$n=1,2, \cdots$, all belong to $C(X)$. To show that this countable set distinguishes points of $X$, we take $x, y \in X$ with $x \neq y$, and note that there is a natural number $n$ and an $\alpha \in A_{n}$ such that $x \in B_{n \alpha}$ and $y \in X \backslash B_{n \alpha}$. If $f_{n}(x)=f_{n}(y)$, then there exists a $\beta \in A_{n}$ such that $y \in B_{n \beta}$ and $\phi_{n \alpha}(x)=$ $\phi_{n \beta}(y)$. Since $\alpha \neq \beta$, however, $f\left(x_{n \alpha}\right) \phi_{n \alpha}(x) \neq f\left(x_{n \beta}\right) \phi_{n \beta}(y)$ whereby $g_{n}(x) \neq$ $g_{n}(y)$, and the proof is complete. 
3.3. Corollary. If $Y$ is any topological space, then $Y$ is separably submetrizable if and only if $Y$ is submetrizable and $\operatorname{card}(Y) \leqq \operatorname{card}(\boldsymbol{R})$.

4. Implications for the strict topology and other observations. The question of separability in Buck's original strict topology is now essentially settled, but this is not the case for the generalized strict topology $\beta$ introduced by Sentilles. For one thing, there is no Stone-Weierstrass type theorem for $\left(C_{b}(X), \beta\right)$ at the present time (except when $\left.\beta=\beta_{0}\right)$, and so an approach similar to the one used for $\beta_{0}$ is not currently available. Nonetheless some progress, though modest, can be made on the problem.

Since $\beta$ is always at least as fine as $\beta_{0}$ [13], it follows from Theorem 2.1 that $X$ must be separably submetrizable whenever $\left(C_{b}(X), \beta\right)$ is separable, but we do not know if the converse is also true. Furthermore, the theorem below shows that no new information can be obtained by applying our results for $\beta_{0}$ in the most obvious way.

4.1. THEOREM. If $\left(C_{b}\left(X_{Q}\right), \beta_{0}\right)$ is separable for some $Q \in \mathscr{2}$, then $X=X_{Q}$, whence $X$ is locally compact.

Proof. Every point in $X_{Q}$ is a $G_{\delta}$ in $\beta X$ since $X_{Q}$ is submetrizable by Theorem 2.1, and thus $X_{Q} \cap(\beta X \backslash X)$ is empty [5, p. 132].

The dual space of $\left(C_{b}(X), \beta\right)$ is the space $\mathscr{M}_{\tau}(X)$ of (complex) $\tau$-smooth (or $\tau$-additive) linear functionals on $C_{b}(X)$ [13, Theorem 4.4], while the linear subspace $\mathscr{M}_{t}(X)$ of $\mathscr{M}_{\tau}(X)$ consisting of the tight linear functionals is the dual space of $\left(C_{b}(X), \beta_{0}\right)[11, \mathrm{p} .41]$. It follows from the work of Knowles [8, p. 148] and Sentilles [13, Theorem 4.2] that if $X$ is absolutely Borel measurable in $\beta X$ (see [8, p. 147]), then $\mathscr{M}_{\tau}(X)=\mathscr{M}_{t}(X)$, and since it is immediate from the Hahn-Banach theorem that a Hausdorff locally convex space is separable if (and only if) it is weakly separable, Theorem 2.1 yields the next result.

4.2. THEOREM. If $X$ is separably submetrizable and absolutely Borel measurable in $\beta X$, then $\left(C_{b}(X), \beta\right)$ is separable.

We are prompted to ask at this point if perhaps separability in the strict topology is sufficient to imply that every $\tau$-smooth functional is tight. $^{2}$ It also seems relevant to note that Theorem 4.2 applies to a fairly extensive class of spaces. In fact, since a Borel set in $\beta X$ is absolutely Borel measurable in $\beta X$, it is easy to see that this class includes the locally compact and the $\sigma$-compact separably submetrizable spaces (the rationals, for example), as well as the topologically complete metric spaces with cardinality not greater than $\operatorname{card}(\boldsymbol{R})$.

\footnotetext{
${ }^{2}$ In an example communicated to the author, R. F. Wheeler has shown this need not be the case.
} 
Each of the spaces $\mathscr{M}_{t}(X)$ and $\mathscr{M}_{\tau}(X)$ may be endowed with the weak star topology from their pairing with $C_{b}(X)$, and any reference to these spaces of a topological nature will henceforth refer to this topology. The following theorem is in analogy with Varadarajan's results on metrizability [17, pp. 188, 192].

4.3. THEOREM. $\mathscr{M}_{t}(X)$ is separably submetrizable if and only if $X$ is separably submetrizable, and either of these conditions is equivalent to the separability of $\left(C_{b}(X), \beta_{0}\right)$.

Proof. It readily follows from [17, p. 187] that $X$ is separably submetrizable whenever $\mathscr{M}_{t}(X)$ has this property. In view of Theorem 2.1, it will suffice to show that if $\left\{f_{k}\right\}_{k=1}^{\infty}$ is $\beta_{0}$ dense in $C_{b}(X)$, then $\mathscr{M}_{t}(X)$ is separably submetrizable. To do this, let $N$ denote the natural numbers with the discrete topology and define a mapping $T$ from $\mathscr{M}_{t}(X)$ into the separable metric space $(C(N), \kappa)$ by $T \mu(k)=\left\langle f_{k}, \mu\right\rangle, \mu \in \mathscr{M}_{t}(X), k \in N$. Since $T$ is clearly one-to-one and continuous, the proof is complete.

A straightforward modification of the above proof shows that if $\left(C_{b}(X), \beta\right)$ is separable, then $\mathscr{M}_{\tau}(X)$ is separably submetrizable (whereby $X$ is separably submetrizable), but even here we do not know if the converse is valid.

There has been some progress on the problem of determining when the space $\left(C_{b}(X), \beta\right)$ is fully complete (e.g., see [14]), but even in the locally compact case there is still only little known. Indeed, the problem is not even resolved when $X=\boldsymbol{R}$, and this leads to our concluding observation.

4.4. THEOREM. If $X$ is a locally compact metrizable space with $\operatorname{card}(X) \leqq$ $\operatorname{card}(\boldsymbol{R})$, then $\left(C_{b}(X), \beta\right)$ is fully complete (has the Krein-Smulian property) if and only if every sequentially closed linear subspace (convex subset) of $\mathscr{M}_{t}(X)$ is closed.

Proof. A well known theorem due to Dieudonné ([17, p. 198], for example) implies $\left(C_{b}(X), \beta\right)$ is sequentially barrelled; i.e., every sequence in $\mathscr{M}_{t}(X)$ which converges to zero is equicontinuous. Since $\left(C_{b}(X), \beta\right)$ is separable by Theorem 3.2, the conclusion is immediate from [19, p. 357].

\section{REFERENCES}

1. R. C. Buck, Bounded continuous functions on a locally compact space, Michigan Math. J. 5 (1958), 95-104. MR 21 \#4350.

2. H. S. Collins, On the space $l^{\infty}(S)$ with the strict topology, Math. Z. 106 (1968), 361-373. MR 39 \#763.

3. J. Dieudonné, Sur un espace localement compact non métrisable, An. Acad. Brasil. Ci. 19 (1947), 67-69. MR 8, 594.

4. R. Giles, A generalization of the strict topology, Trans. Amer. Math. Soc. 161 (1971), 467-474. 
5. L. Gillman and M. Jerison, Rings of continuous functions, University Series in Higher Math., Van Nostrand, Princeton, N.J., 1960. MR 22 \#6994.

6. J. G. Hocking and G. S. Young, Topology, Addison-Wesley, Reading, Mass., 1961. MR 23 \#A2857.

7. J. L. Kelley, General topology, Van Nostrand, Princeton, N.J., 1955. MR 16, 1136.

8. J. D. Knowles, Measures on topological spaces, Proc. London Math. Soc. (3) 17 (1967), 139-156. MR 34 \#4441.

9. L. LeCam, Convergence in distribution of stochastic processes, Univ. Calif. Publ. Statist. 2 (1957), 207-236. MR 19, 128.

10. A. P. Robertson and W. J. Robertson, Topological vector spaces, Cambridge Tracts in Math. and Math. Phys., no. 53, Cambridge Univ. Press, New York, 1964. MR 28 \#5318.

11. A. C. M. van Rooij, Tight functionals and the strict topology, Kyungpook Math. J. 7 (1967), 41-43. MR 37 \#3336.

12. Z. Semadeni and P. Zbijewski, Spaces of continuous functions. I, Studia Math. 16 (1957), 130-141. MR 19, 1182.

13. F. D. Sentilles, Bounded continuous functions on a completely regular space, Trans. Amer. Math. Soc. 168 (1972), 311-336.

14. W. H. Summers, Full-completeness in weighted spaces, Canad. J. Math. 22 (1970), 1196-1207.

15. - The general complex bounded case of the strict weighted approximation problem, Math. Ann. 192 (1971), 90-98.

16. C. Todd, Stone-Weierstrass theorems for the strict topology, Proc. Amer. Math. Soc. 16 (1965), 654-659. MR 31 \#3891.

17. V. S. Varadarajan, Measures on topological spaces, Mat. Sb. 55 (97) (1961), 35-100; English transl., Amer. Math. Soc. Transl. (2) 48 (1965), 161-228. MR 26 \#6342.

18. S. Warner, The topology of compact convergence on continuous function spaces, Duke Math. J. 25 (1958), 265-282. MR 21 \#1521.

19. J. H. Webb, Sequential convergence in locally convex spaces, Proc. Cambridge Philos. Soc. 64 (1968), 341-364. MR 36 \#5652.

Department of Mathematics, University of Arkansas, Fayetteville, Arkansas 72701 